\title{
Aspectos epidemiológicos do câncer infantojuvenil em uma capital do nordeste brasileiro
}

\author{
Epidemiological aspects of childhood cancer in a capital of northeast Brazil
}

Aspectos epidemiológicos del cáncer infantil en una capital del nordeste de Brasil

Walter Marcelo Oliveira de Carvalho ${ }^{1}$, Rute Nascimento da Silva ${ }^{1}$, Marcela Beatriz Feitosa de Carvalho $^{1}$, Thaynara Ferreira Batista ${ }^{1}$, Renata Isabela Feitosa de Carvalho Nascimento ${ }^{1}$, Marcos Vinicius Ribeiro Nascimento ${ }^{1}$, Carla Viviane Freitas de Jesus ${ }^{1 *}$, Verônica de Lourdes Sierpe Jeraldo ${ }^{1}$.

\section{RESUMO}

Objetivo: Objetivou-se caracterizar os aspectos epidemiológicos e analisar a curva de sobrevivência e razão de risco do câncer infantojuvenil em uma capital do nordeste brasileiro. Métodos: Constitui-se em estudo transversal, exploratório, retrospectivo, de abordagem quantitativa, realizado no Registro de Câncer de Base Populacional (RCBP), abrangendo crianças e adolescentes, entre 0 a 19 anos, portadoras de neoplasias malignas, selecionaram-se 515 casos. Resultados: Os grupos de neoplasias malignas mais incidentes foram respectivamente: o grupo XI (outras neoplasias malignas), o grupo I (leucemias); o grupo II (linfomas) e o grupo III (tumores do SNC). O sexo feminino obteve maiores percentuais dentre os acometidos, notadamente nos grupos XI (Outras neoplasias malignas epiteliais), I (Leucemias), II (Linfomas) e III (Tumores do SNC). No masculino, destacaram-se os grupos I, II, III e XI. Conclusão: Observou-se que o exame diagnóstico mais utilizado foi a histologia do tumor primário. Ao analisar a curva de sobrevida se constatou que é menor no sexo feminino e nas faixas etárias de $<1$ ano, 1 a 4,5 a 9, e 10 a 14 anos do que a faixa de 15 a 19 anos.

Palavras-chave: Neoplasias, Epidemiologia, Criança, Adolescente.

\begin{abstract}
Objective: This study aimed to characterize the epidemiological aspects and to analyze the survival curve and risk ratio of childhood cancer in a capital of northeastern Brazil. Method: It is a cross-sectional, exploratory, retrospective study. of quantitative approach, carried out in the Population-Based Cancer Registry (RCBP), covering children and adolescents, aged 0 to 19 years, with malignant neoplasms, a total of 515 cases. Results: The most incident malignant groups were: XI (other malignant neoplasms), group I (leukemias); group II (lymphomas) and group III (CNS tumors). Female gender obtained higher percentages among the affected ones, notably in groups XI (Other epithelial malignant neoplasms), I (Leukemias), II (Lymphomas) and III (CNS Tumors). In males, groups I, II, III and XI stood out. Conclusion: It was observed that the most used diagnostic test was histology of the primary tumor. When analyzing the survival curve, it was found that it is lower in females and in the age groups $<1$ year, 1 to 4,5 to 9 , and 10 to 14 years than the 15 to 19 age group.
\end{abstract}

Keywords: Neoplasms, Epidemiology, Children, Teen.

\section{RESUMEN}

Objetivo: Este estudio tuvo como objetivo caracterizar los aspectos epidemiológicos y analizar la curva de supervivencia y la relación de riesgo de cáncer infantojuveil en una capital del noreste de Brasil. Métodos: Es un estudio transversal, exploratorio, retrospectivo. de enfoque cuantitativo, llevado a cabo en el Registro de cáncer de base poblacional (RCBP), que abarca niños y adolescentes, de 0 a 19 años, con neoplasias malignas, un total de 515 casos. Resultados: Los grupos malignos más incidentes fueron: XI (otras neoplasias malignas), grupo I (leucemias); grupo II (linfomas) y grupo III (tumores del SNC). El género femenino obtuvo porcentajes más altos entre los afectados, especialmente en los grupos XI (Otras neoplasias malignas epiteliales), I (Leucemias), II (Linfomas) y III (Tumores del SNC). En los varones, se destacaron los

1 Universidade Tiradentes (UNIT), Aracaju - SE. *E-mail: carlavfj@gmail.com 
grupos I, II, III y XI. Conclusión: Se observó que la prueba diagnóstica más utilizada fue la histología del tumor primario. Al analizar la curva de supervivencia, se encontró que es menor en el sexo femenino y en los grupos de edad <1 año, 1 a 4, 5 a 9 y 10 a 14 años que el grupo de 15 a 19 años.

Palabras clave: Neoplasias, Epidemiología, Niños, Adolescente.

\section{INTRODUÇÃO}

O câncer infantojuvenil (CIJ) corresponde a um grupo de doenças que têm em comum a proliferação descontrolada de células anormais que pode ocorrer em qualquer topografia do organismo (CURVO HRM, et al., 2013). Doenças malignas da infância afetam as células do sistema hematopoiético e os tecidos de sustentação, porque são predominantemente de natureza embrionária, oriundos de células indiferenciadas, enquanto o câncer do adulto afeta as células do epitélio (HANNA N, et al., 2017).

O ClJ é considerado raro quando comparado com os tumores do adulto, correspondendo entre 2 e $3 \%$ de todas as neoplasias malignas. Varia de acordo com o tipo histológico, localização primária do tumor, etnia, sexo e idade (CURVO HRM, et al., 2013; PESOLA F, et al., 2017). Geralmente apresentam curtos períodos de latência, são mais agressivos e crescem mais rapidamente. No entanto, respondem melhor aos tratamentos, configurando-se com um melhor prognóstico. Resulta em desgaste psíquico, social e financeiro, conferindo um impacto profundo nos pacientes, famílias, sociedade e no sistema público de saúde, representando importante perda de potenciais anos de vida (CURVO HRM, et al., 2013; HANNA N, et al., 2017).

Informações sobre ClJ e subtipos de câncer por fatores demográficos e temporais são escassos (SIEGEL DA, et al., 2014). A epidemiologia descritiva do câncer é indispensável fonte de informação. Não só oferece conhecimento das etiologias destas doenças, mas também contribui como um indicador do progresso das políticas de saúde pública (BRAVO LE, et al., 2013).

O conhecimento da incidência de câncer é importante na identificação de risco nas populações, bem como para esclarecer o papel potencializador dos fatores ambientais, estabelecer provisão de serviços, definir linha de base para avaliar a eficácia de intervenções, seu impacto no cuidado e na sobrevivência, alocar recursos e no desenvolvimento de pesquisas (MARQUANT F, et al., 2016; PESOLA F, et al., 2017). Mudanças de incidências incluem variações em capacidade diagnóstica, completude de registro, composição populacional e exposição a fatores de risco (STELIAROVA-FOUCHER E, et al., 2018).

De acordo com Franco EL (1994), poucos países possuem sistemas de monitoramento com cobertura para toda a população, com isso os registros de câncer, na maioria das vezes se limitam a certas cidades, 0 que contribui para que os dados sobre a doença não reflitam a realidade da saúde. Portanto o presente estudo objetivou caracterizar os aspectos epidemiológicos assim como analisar a curva de sobrevivência e razão de risco do ClJ no município de Aracaju, Estado de Sergipe, entre os anos 1996 e 2014.

\section{MÉTODOS}

Trata-se de um estudo transversal, de caráter exploratório, retrospectivo, de abordagem quantitativa, realizado em banco de dados cadastrais, no Registro de Câncer de Base Populacional (RCBP), localizado no Centro de Oncologia do hospital referência do estado avaliado, abrangendo crianças e adolescentes, entre 0 a 19 anos, portadoras de neoplasias malignas.

Dentre os 12 tipos listados na Classificação Internacional de Câncer Infantil (CICl) o Instituto Nacional do Câncer (INCA) estimou para o Estado de Sergipe, em 2018, 80 casos novos de CIJ para o sexo masculino e 95 casos para o feminino (SES-SE, 2018).

$\mathrm{O} \mathrm{CICl}$ inclui 12 grupos tumorais principais e 47 subgrupos incluindo apenas tumores considerados malignos, com exceção do grupo III, onde também estão inseridos tumores intracranianos ou intra-espinhais de histologia benigna. Os 12 grupos principais são: I leucemias, II linfomas e neoplasmas reticulares, III tumores do SNC, IV neuroblastoma e outros tumores de células nervosas periféricas, V retinoblastoma, VI 
tumores renais, VII tumores hepáticos, VIII tumores ósseos malignos, IX sarcomas de tecido mole, X tumores germinativos, trofoblásticos e outros tumores gonadais, XI outra neoplasia epitelial e melanoma e XII outras neoplasias não especificadas (BRAVO LE, et al., 2013).

No estudo foram incluídas todas as crianças e adolescentes, entre 0 a 19 anos em tratamento de neoplasias malignas, cadastrados no RCBP, residentes no município estudado, uma capital do nordeste brasileiro. Selecionaram-se 515 casos, entre 1996 a 2014, excluindo-se aqueles com registros do tipo de câncer ignorados no banco de dados.

Considerou-se os casos de CIJ de localização primária determinada, os de localização secundária e os de localização incerta (se primários ou metastáticos), os casos de bases diagnósticas por estudos histológicos, citológicos, hematológicos, exploração cirúrgica, imagem, exame clínico ou qualquer outro meio de diagnóstico, efetuado pelo médico responsável, e aqueles cuja modalidade de registro ocorreu pela Declaração de Óbito (SDO), nos casos que não puderam ser identificados em outras fontes ou bases de dados.

As variáveis foram descritas por meio de frequência absoluta e relativa percentual. Realizou-se a caracterização exploratória das variáveis dividindo a amostra por cada categoria da $\mathrm{ClCl}$. A sobrevida foi estimada por meio do estimador de Kaplan-Meier. As razões de risco bruta foram calculadas por meio da regressão de Cox. O nível de significância adotado foi de 5\% e o software utilizado foi o R Core Team 2019. O presente estudo foi aprovado pelo Comitê de Ética e Pesquisa da Universidade Tiradentes, sob o parecer consubstanciado ํㅜ 3.239.059.

\section{RESULTADOS}

Observou-se que os grupos de ClJ mais frequentes no período analisado foram respectivamente: o grupo XI (outras neoplasias malignas e outros melanomas malignos), correspondendo a $24,85 \%$ (128); grupo I (leucemias, doenças mieloproliferativas e doenças mielodisplásicas) com 18,25\% (94); grupo II (linfomas e doenças reticuloendoteliais) com 16,5\% (85) e o grupo III (tumores do sistema nervoso central (SNC) e miscelânea de neoplasias intracranianas e intraespinhais), com 13,98\% (72) (Tabela 1).

Tabela 1 - Frequências dos cânceres infantojuvenis de acordo com a $\mathrm{CICl}$ em uma capital do nordeste brasileiro, $(n=515)$.

\begin{tabular}{ccc}
\hline CICl & $\mathbf{n}$ & $\%$ \\
\hline I(Leucemias) & 94 & 18,25 \\
II(Linfomas) & 85 & 16,5 \\
III(Tumores do sistema nervoso central) & 72 & 13,98 \\
IV(Neuroblastomas) & 17 & 3,30 \\
V(Retinoblastomas) & 07 & 1,36 \\
VI(Tumores Renais) & 18 & 3,5 \\
VII(Tumores Hepáticos) & 09 & 1,75 \\
VIII(Tumores ósseos) & 26 & 5,05 \\
IX(Tecidos moles e outros sarcomas) & 32 & 6,21 \\
X(Tumores de células germinativas) & 24 & 4,66 \\
XI(Outras neoplasias malignas epiteliais) & 128 & 24,85 \\
XII(Outras neoplasias malignas não especificadas) & 03 & 0,58 \\
\hline Total & 515 & 100,0
\end{tabular}

Fonte: Carvalho WMO, et al., 2020.

Com relação ao sexo dos acometidos, foram observados maiores percentuais de $\mathrm{ClJ}$ no sexo feminino $(54,31 \%)$ que no masculino $(45,69 \%)$. No feminino, registraram-se, maiores percentuais, respectivamente, nos grupos XI (Outras neoplasias malignas epiteliais), I (Leucemias), II (Linfomas) e III (Tumores do SNC). No masculino, destacaram-se, em ordem decrescente, os grupos I, II, III e XI (Figura 1). 
Figura 1 - Cânceres infantojuvenis por gênero em uma capital do nordeste brasileiro.

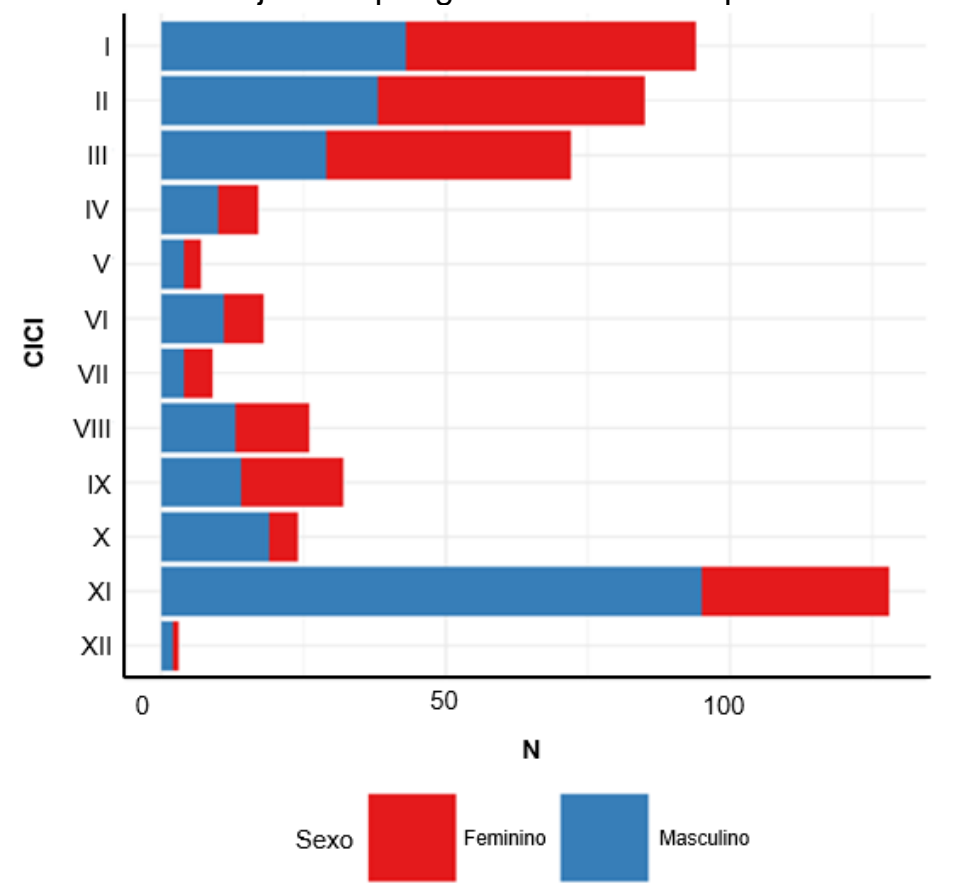

Fonte: Carvalho WMO, et al., 2020.

No que se refere as faixas etárias, os maiores percentuais dos portadores de CIJ, estão, respectivamente, com idades compreendidas entre 15 a 19 anos (42,38\%), principalmente no grupo XI (outras neoplasias malignas epiteliais) e no grupo II (linfomas), e entre 1 a 4 anos (19,80\%), no qual predominou o grupo I (leucemias) e o grupo III (Tumores do SNC). A menor frequência foi registrada em menores de 01 ano, resultando em 3,37\%, destacando- se principalmente o grupo I (leucemias) e os grupos III e IV (tumores do SNC e neuroblastomas). Deve-se registrar que os grupos I (leucemias), III (tumores do SNC), IV (neuroblastomas), IX (tecidos moles e outros sarcomas) e X (tumores de células germinativas) registraram casos em todas as faixas etárias estudadas (Figura 2).

Figura 2 - Cânceres infantojuvenis por faixa etária em uma capital do nordeste brasileiro.

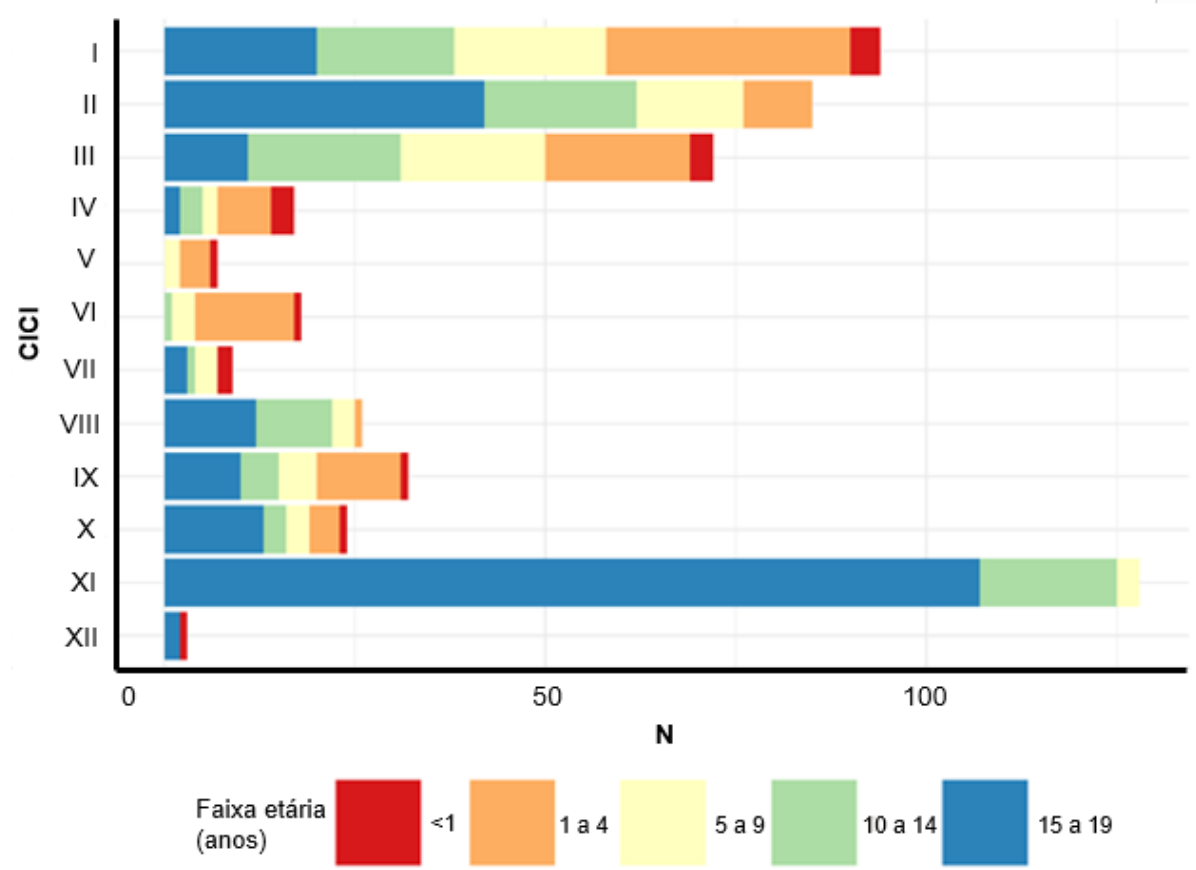

Fonte: Carvalho WMO, et al., 2020. 
Segundo a cor, observou-se que os dados ignorados atingiram maiores percentuais nas classificações XI, I, II, III, VI, X e VII. A cor registrada com maiores percentuais em todas as classificações ocorreu em pardos. Ao analisar a classificação dos grupos de neoplasias se pode perceber que no grupo I, $71,3 \%$ (67) dos casos registrados de leucemias, doenças mielodisplásicas e doenças mieloproliferativas, corresponderam à classificação $\mathrm{A}$, ou seja, segundo a $\mathrm{CICl}$, às leucemias linfóides.

No grupo II, que congrega os linfomas e neoplasias reticuloendoteliais, prevaleceu a classificação B, categorizados como linfomas não Hodgkin. No grupo III (tumores do SNC e miscelâneas de neoplasias intracranianas e intraespinhais), destacou-se a classificação $F$ (neoplasias intracranianas e intra-espinhais não especificadas).

No grupo IV o maior percentual ficou concentrado na classificação $B$ (neuroblastomas). O grupo $V$ é único, representado apenas pelos retinoblastomas. O grupo VI (tumores renais) teve maior percentual de nefroblastoma (classificação A). No VII (tumores hepáticos) preponderaram os hepatoblastomas (classificação A).

No grupo VIII (tumores ósseos malignos) também prevaleceu a classificação A (osteossarcomas). No IX (tecidos moles e outros sarcomas extraósseos), também a classificação $A$ (rabdomiossarcomas). No grupo $X$ (tumores de células germinativas), a classificação C (tumores malignos de células germinativas gonadais).

No grupo XI (outros neoplasmas malignos epiteliais) destacou-se a classificação $F$ (outros carcinomas e carcinomas não especificados). No grupo XII (Outras neoplasias malignas não especificadas) todos os casos se concentram na classificação B que corresponde a outros tumores malignos não especificados (Figura 3).

Figura 3 - Cânceres infantojuvenis por classificação dos grupos de neoplasias em uma capital do nordeste brasileiro.

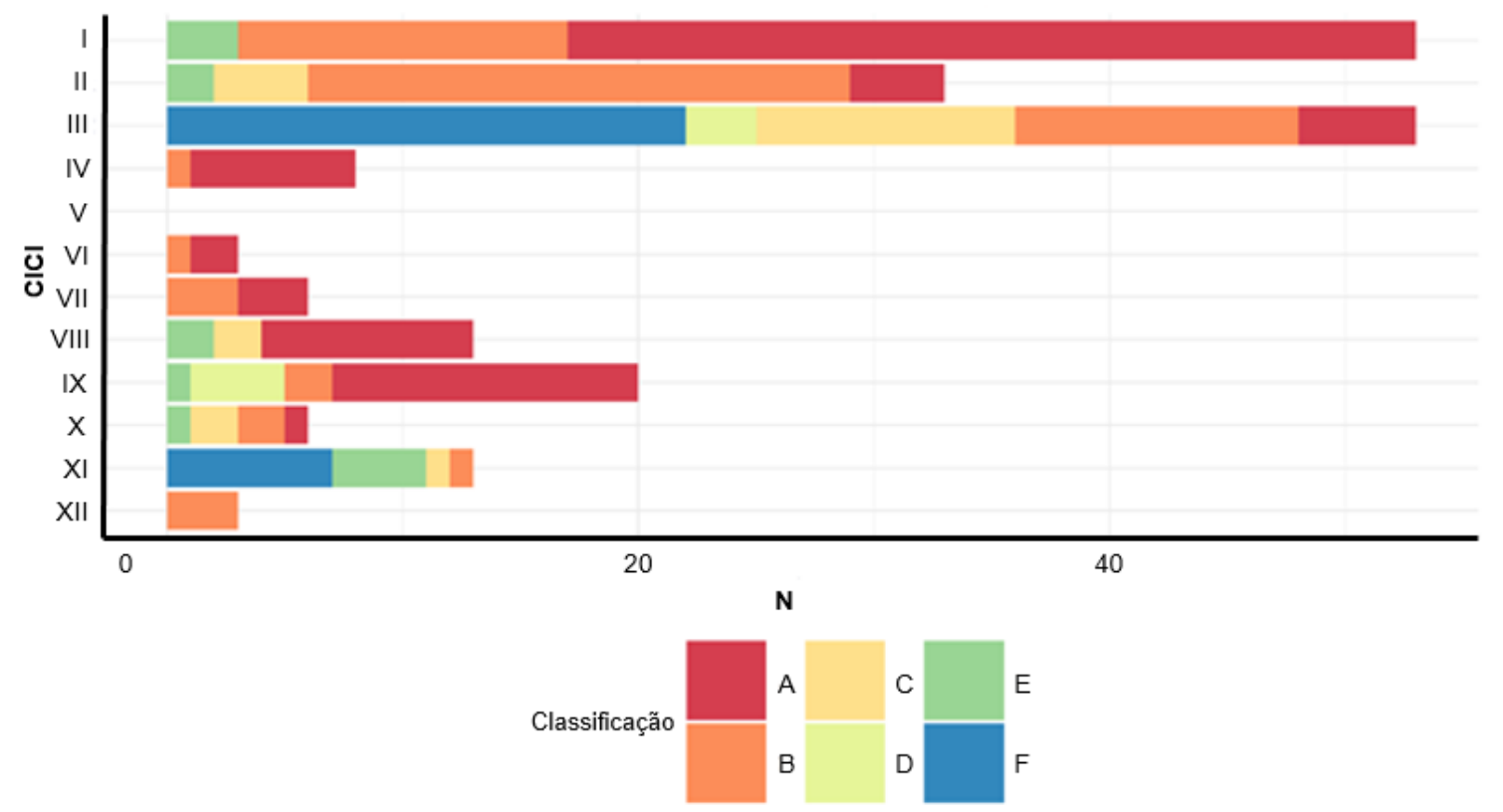

Fonte: Carvalho WMO, et al., 2020.

Nos grupos II, III, IV, V, VI, VII, VIII, IX, X e XI o exame diagnóstico mais utilizado foi a histologia do tumor primário. No Grupo I o maior percentual se concentrou no diagnóstico citológico e no grupo XII as classificações foram realizadas por declaração de óbitos (SDO) e por Pesquisa Clínica.

Ao analisar a curva de sobrevida estimada (Kaplan-Meier) para os pacientes em 5 anos, observou-se que $61,2 \%$ dos acometidos continuam vivos. As figuras 4(a), 4(b), 4(c) e 4(d) apresentam as curvas de sobrevida estratificadas por sexo, faixa etária e $\mathrm{CICl}$. Pode-se observar que a sobrevida é menor entre crianças do sexo feminino, de menor faixa etária, notadamente nas Leucemias, Linfomas, Tumores do SNC, Neuroblastomas, Retinoblastomas, Tumores Hepáticos, Tumores Ósseos, Sarcomas de tecido mole, Tumores nas células germinativas e Outras neoplasias não especificada (Figura 4). 
Figura 4 - Curva de sobrevida estimada em 5 anos por sexo, faixa etária e classificação dos grupos de neoplasias, dos cânceres infanto-juvenis em uma capital do nordeste brasileiro.

(A)

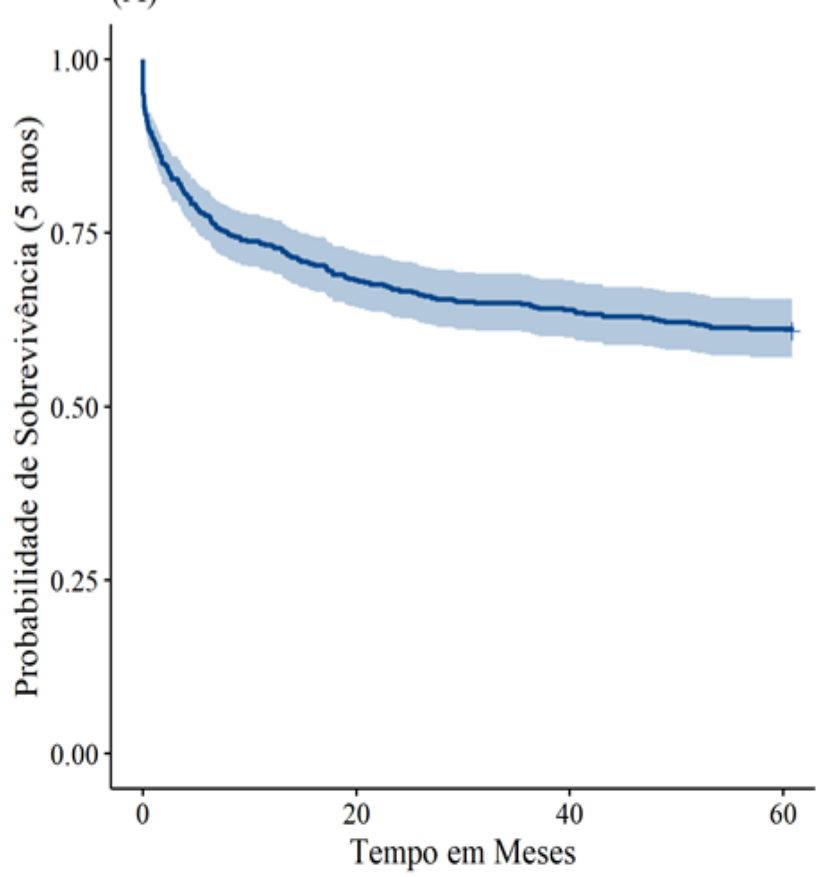

(B)

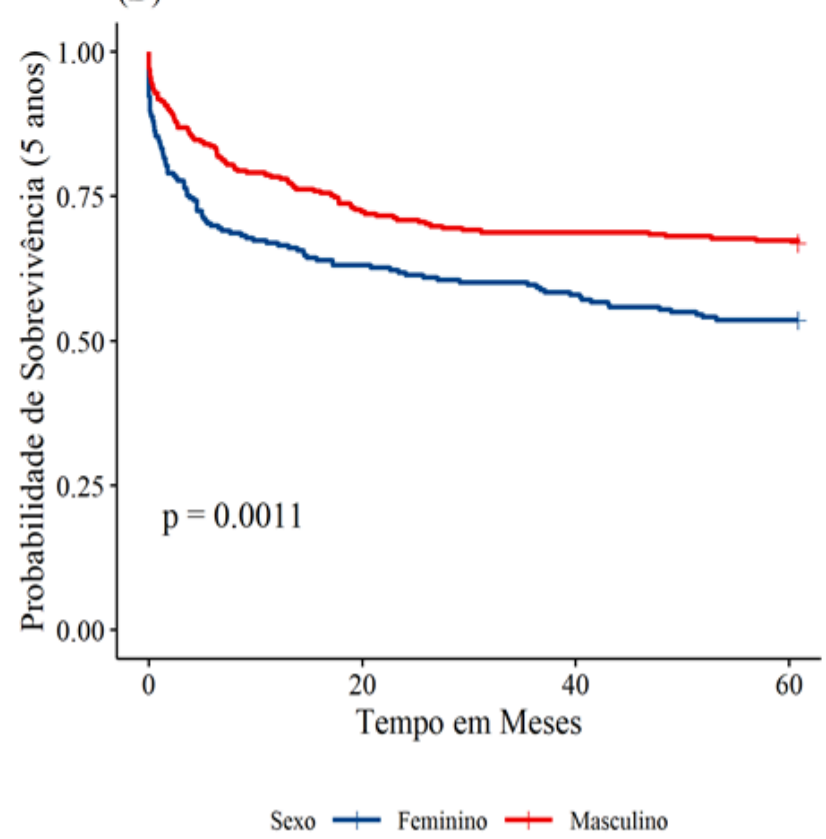

(C)

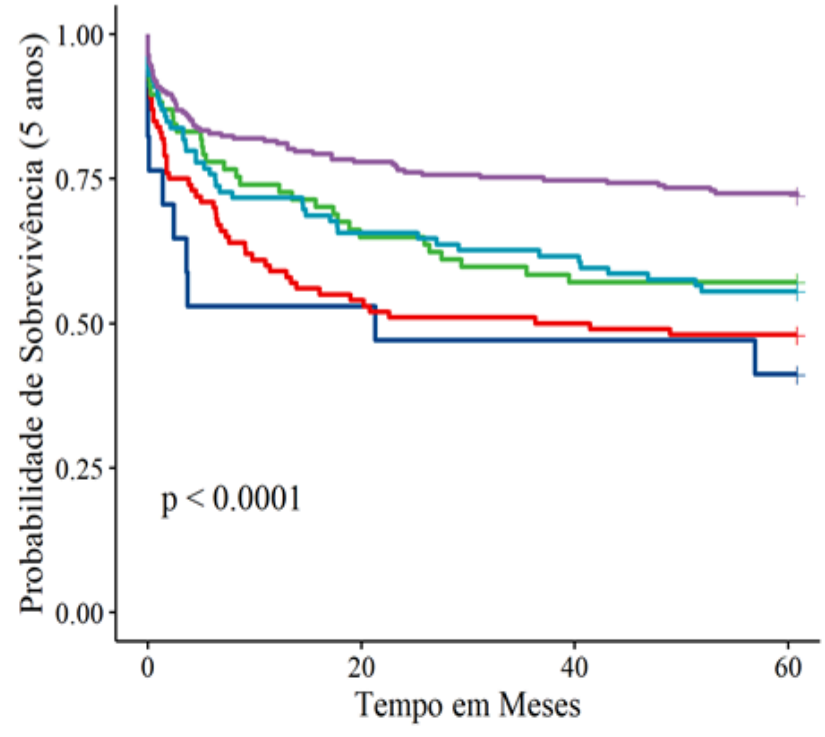

Faixa Etária $+<1 \div 1-4 \div 5-9 \div 10-14 \div 15-19$

(D)

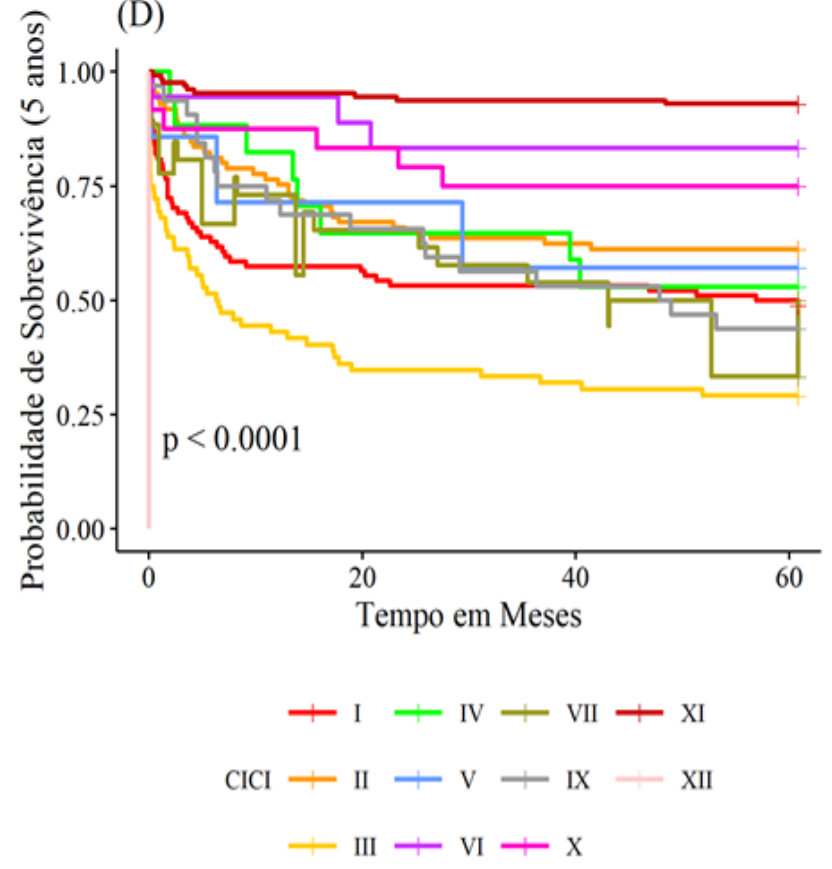

Fonte: Carvalho WMO, et al., 2020.

Ao analisar as razões de risco para sexo, faixa etária e $\mathrm{CICl}$, pode-se observar entre os resultados significativos que o sexo feminino apresentou maior risco de óbito em 5 anos do que o masculino, as faixas etárias de <1 ano, 1 a 4 anos, 5 a 9 anos, 10 a 14 anos apresentaram maior risco de óbito em 5 anos do que a faixa de 15 a 19 anos e quanto a $\mathrm{CICl}$, Leucemias, Linfomas, Tumores do SNC, Neuroblastomas, Retinoblastomas, Tumores Hepáticos, Tumores Ósseos, Sarcomas de tecido mole, Tumores nas células germinativas e Outras neoplasias não especificadas apresentaram maior risco de óbito em 5 anos do que Outras Neoplasias Malignas Epiteliais. Apenas os tumores renais não apresentaram maior risco de morte em 5 anos (Tabela 2). 
Tabela 2 - Razões de risco para sexo, faixa etária e Classificação Internacional de câncer infantojuvenil em uma capital do nordeste brasileiro.

\begin{tabular}{cccc}
\hline Sexo & RR & IC95\% & p-valor \\
\hline Feminino & & & \\
Masculino & 1,59 & $1,20-2,08$ & 0,001 \\
\hline Faixa Etária & 1 & & \\
\hline 1 1 ano & & & \\
5 a a 9 anos & 2,85 & $1,46-5,57$ & 0,002 \\
10 a 14 anos & 2,26 & $1,56-3,26$ & $<0,001$ \\
15 a 19 anos & 1,66 & $1,09-2,54$ & 0,018 \\
CICl & 1,74 & $1,18-2,56$ & 0,005 \\
\hline I(Leucemias) & 1 & & \\
II(Linfomas) & & & $<0,001$ \\
III (Tumores do SNC) & 10,36 & $5,08-21,13$ & $<0,001$ \\
IV(Neuroblastomas) & 6,63 & $3,17-13,85$ & $<0,001$ \\
V(Retinoblastomas) & 17,93 & $8,82-36,49$ & $<0,001$ \\
VI (Tumores Renais) & 7,86 & $3,03-20,39$ & 0,003 \\
VII (Tumores Hepáticos) & 7,49 & $2,03-27,65$ & 0,175 \\
VIII (Tumores ósseos) & 2,47 & $0,67-9,12$ & $<0,001$ \\
IX (Tecidos moles/sarcomas) & 13,10 & $4,66-36,83$ & $<0,001$ \\
X (Tumores células germinativas) & 9,00 & $3,84-21,05$ & $<0,001$ \\
XII (Outras neo malignas não especificadas) & 9,81 & $4,40-21,84$ & 0,009 \\
XI (Outras neo malignas epiteliais) & 171,65 & $1,41-11,09$ & $<0,001$ \\
\hline
\end{tabular}

Fonte: Carvalho WMO, et al., 2020.

\section{DISCUSSÃO}

No presente estudo, excluindo-se o Grupo XI que concentra uma miscelânia de outras neoplasias malignas epiteliais, os CIJ mais incidentes foram as leucemias, linfomas e tumores do SNC. Outros estudiosos também registraram estudos com as maiores frequências de $\mathrm{ClJ}$ em leucemias, doenças mieloproliferativas e mielodisplásicas (FREITAS VA, et al., 2013). As leucemias foram as neoplasias pediátricas mais comuns, seguidas pelos tumores do SNC e os linfomas, coincidindo com os resultados aqui encontrados (DINIZ AB, et al., 2005; PEDROSA MF, et al., 2007). Neste sentido, as leucemias e os tumores do SNC correspondem a metade de todos os cânceres que incidem nessa faixa etária, para outros autores as leucemias constituem o maior grupo de neoplasias na infância, seguidas pelos tumores do SNC que se constituem no maior grupo de tumores sólidos infantis (TEIXEIRA AB, 2017).

Concordando com os resultados dessa pesquisa alguns autores observaram que a incidência do Grupo IX (Tecidos moles/sarcomas), em crianças e adolescentes, representou 7,4\% de todas as neoplasias nessa faixa etária, oscilando entre $4 \%$ a $8 \%$ de todas as neoplasias malignas da infância (FRIESTINO JKO, 2017).

A distribuição dos casos de CIJ, no que se refere ao sexo dos acometidos, foi maior no sexo feminino, resultado coincidente com aquele descrito por Santos CJ, et al. (2018), uma vez que verificaram uma frequência mundial de casos de ClJ variando de 232,31 por milhão no sexo feminino e 218,07 por milhão para o sexo masculino, assim como pesquisas apontaram carcinomas e outras neoplasias malignas epiteliais, e nos tumores renais, ocorreram também com maior frequência no sexo feminino (BOUZAS LF e CALAZANS M, 2007).

No que se refere aos tumores de células germinativas, a maior frequência aqui encontrada foi no sexo feminino, divergindo daqueles relatados por Rondinelli PI, et al. (2005), uma vez que encontraram este grupo de neoplasias principalmente no sexo masculino. Fato semelhante ocorreu com referência aos neuroblastomas (BOUZAS LF e CALAZANS M, 2007; LUCENA JN, et al., 2018) descreveram maior frequência no sexo masculino, enquanto neste estudo a maior incidência ocorreu no sexo feminino. Também houve divergência nos tumores ósseos, uma vez que aqui foram mais frequentes no sexo masculino e para Souza MHG, et al. (2019) o Sarcoma de Ewing, por exemplo, é uma neoplasia óssea mais incidente no sexo feminino. 
Com relação a distribuição do CIJ por faixas etárias a maior frequência aqui determinada ficou concentrada no grupo compreendido entre 15 a 19 anos, concordando com os resultados apresentados por Balmant NV, et al. (2019) que registraram que mundialmente o ClJ varia de 100 a 180 por milhão de crianças menores de 15 anos e 210,42 por milhão no grupo de adolescentes entre 15 e 19 anos. Em confronto com os resultados relatados nesta pesquisa, para Teixeira AB (2017) a incidência do ClJ é maior no primeiro ano, apresentando um segundo pico entre o segundo e terceiros anos de vida.

Neste estudo, entre 15 e 19 anos prevaleceu, excluindo-se o grupo de outras neoplasias malignas epiteliais, os linfomas, leucemias, tumores de células germinativas, tumores ósseos e tumores do SNC. Também para Aristizabal-Arboleda LP (2018) nos pacientes de 15-19 anos, os linfomas também representaram as neoplasias mais frequentes, seguido de tumores do SNC, leucemia e tumores de células germinativas. Por outro lado, discordando dos resultados aqui apresentados, Santos CJ, et al. (2018) afirmaram que a faixa etária de menor incidência está situada entre 15 a 19 anos, registrando que crianças de 1 a 4 anos de idade tiveram as maiores taxas de incidência, resultados semelhantes aqueles relatados por Bauer DFV, et al. (2016) em estudo realizado para caracterizar as internações de crianças com câncer, verificando que $43 \%$ tinham entre 1 a 4 anos.

No que se refere aos tumores de tecidos moles e outros sarcomas (Grupo IX), são raros e a incidência predomina em adolescentes. Porém, neste estudo a maior frequência foi encontrada no grupo compreendido entre 1 a 4 anos (FRIESTINO JKO, 2017).

No que se refere à classificação dos tipos de $\mathrm{ClJ}$, a literatura apresenta registros semelhantes aos deste estudo. Aqui foi referenciado que as leucemias foram mais incidentes nas faixas etárias compreendidas entre 0 a 14 anos, atingindo o pico entre 1 a 4 anos, resultados também constatados por Teixeira $A B$ (2017) que afirmou ser a leucemia, na faixa etária compreendida entre 0 e 14 anos, o tumor mais comum, e por outro autor, para os quais, nas leucemias, a faixa etária de 1 a 4 anos, foi a mais acometida.

De acordo com Martins SMG e Lima TNST (2019) entre crianças, os principais tumores do SNC ocorrem principalmente em menores de 15 anos, sendo 10 anos o pico de idade. Aqui, fato semelhante ocorreu, pois os tumores do SNC tiveram maiores e iguais frequências entre 5 a 9 e 10 a 14 anos.

Para Rondinelli PI, et al. (2005), no grupo X (tumores de células germinativas) a média de idade de acometimento também foi na adolescência. Neste estudo a maior frequência dos tumores ósseos ficou concentrada entre 10 a 14 aos, concordando com o relato de Santos CJ, et al. (2018) segundo os quais, na adolescência, o Sarcoma de Ewing tem maior incidência durante a fase da puberdade e o osteossarcoma em crianças com idades que variaram entre 10 a 14 anos.

Argollo N e Lessa I (1999) citaram estudos franceses e suecos nos quais a maior incidência de neoplasias cerebrais na infância estabelecida na faixa etária compreendida entre 0 a 4 anos. Esta constatação foi também encontrada neste estudo quando se procede ao somatório das frequências entre menores de um ano e crianças com idades compreendidas entre 1 a 4 anos.

Dados concordantes com esta pesquisa e a literatura também foram verificados com relação ao neuroblastoma, uma vez que Warlet F, et al. (2018) e Lucena JN, et al. (2018) relataram ser o tumor sólido extracraniano maligno mais comum diagnosticado no primeiro ano de vida. Outro ponto de convergência foi verificado com relação aos tumores hepáticos, pois para Hiyama E (2014) geralmente é diagnosticado durante os primeiros três anos de vida.

Houve relatos de que nas leucemias em crianças, verifica-se que $85 \%$ dos casos, são do tipo linfóide aguda (LLA) o que foi verificado neste estudo. Fato semelhante ocorreu em relação aos linfomas, uma vez que Teixeira $A B$ (2017) observaram que na faixa etária de 0 a 15 anos, $60 \%$ dos casos representados pelos linfomas não-Hodgkin (LNH). (TEIXEIRA AB, 2017; FRIESTINO JKO, 2017).

O método diagnóstico mais utilizado observado neste estudo foi a histologia do tumor primário. AristizabalArboleda LP, (2018) relataram que diagnósticos histopatológicos são importantes para o diagnóstico do linfoma de Burkitt, o linfoma de Hodgkin clássico tipo esclerose nodular, o carcinoma nasofaríngeo e o rabdomiossarcoma. Souza MHG, et al. (2019) o diagnóstico definitivo do sarcoma de Ewing é o exame 
microscópico. Segundo Argollo N e Lessa I (1999) em neoplasia cerebral o diagnóstico definitivo é feito pelo estudo anatomopatológico da peça cirúrgica Hiyama E (2014) destacou que no hepatoblastoma é essencial o diagnóstico histológico de uma amostra tumoral.

No rabdomiossarcoma o diagnóstico requer interpretação da avaliação patológica e o conhecimento do padrão de disseminação metastática. De acordo com Monte O, et al. (2017) nos tumores germinativos o diagnóstico é definido através da biópsia do tumor primário ou de um linfonodo supraclavicular comprometido. (BOUZAS LF e CALAZANS M, 2007).

Neste estudo as razões de risco para óbitos em cinco anos foi maior no sexo feminino e em faixas etárias compreendidas entre 0 a 14 anos. Marchi JÁ, et al. (2013) ao analisar o risco de óbito por câncer infantil relataram que o sexo feminino é mais vulnerável, assim como a faixa etária de 0 a 9 anos, dados que concordam com a presente pesquisa. Cardous-Ubbink MC, et al. (2007) também relataram maior frequência de mortalidade no sexo feminino, porém em faixas etárias compreendidas entre 10 e 16 anos.

Dentre os $\mathrm{CIJ}$ com maior mortalidade destacaram-se as leucemias, linfomas tumores do SNC e Neuroblastomas. Em outro estudo, destacaram que óbitos prevalecendo as leucemias, as neoplasias do SNC e os linfomas. Porém, com relação a faixa etária relataram que adolescentes apresentaram um risco aproximadamente três vezes maior de óbito em comparação com as crianças, aspecto discordante deste estudo (BRAGA PE, et al., 2002). Em estudo de sobrevida realizado no serviço de oncologia pediátrica do Hospital de Clínicas da Universidade Federal do Paraná registraram que em uma amostra pequena, por grupo de neoplasia, não foi possível realizar uma análise minuciosa de outras variáveis (HADAS TC, et al., 2014).

\section{CONCLUSÃO}

Através da caracterização dos aspectos epidemiológicos do ClJ uma capital do nordeste brasileiro, no período de 1996 a 2014, os grupos de neoplasias malignas mais incidentes foram respectivamente: o grupo XI (outras neoplasias malignas), o grupo I (leucemias); o grupo II (linfomas) e o grupo III (tumores do SNC). O sexo feminino obteve maiores percentuais dentre os acometidos, notadamente nos grupos XI (Outras neoplasias malignas epiteliais), I (Leucemias), II (Linfomas) e III (Tumores do SNC). No masculino, destacaram-se, em ordem decrescente, os grupos I, II, III e XI. Quanto as faixas etárias, os maiores percentuais estão, respectivamente, naqueles com idades compreendidas entre 15 a 19 anos, principalmente no grupo XI (outras neoplasias malignas epiteliais) e no grupo II (linfomas), e entre 1 a 4 anos, no grupo I (leucemias) e o grupo III (Tumores do SNC).

\section{REFERÊNCIAS}

1. ARGOLLO N, LESSA I. Aspectos clínico-epidemiológicos das neoplasias cerebrais na faixa etária pediátrica no Estado da Bahia, Brasil. Arquivos de Neuropsiquiatria, 1999; 57(2-B): 442- 451.

2. ARISTIZABAL-ARBOLEDA, LP. Distribuição demográfica e clinicopatológica dos tumores malignos de cabeça e pescoço em pacientes pediátricos de uma instituição brasileira: estudo retrospective. (Mestrado em Estomatopatologia) - Piracicaba: Universidade Estadual de Campinas, 2018, 49p.

3. BALMANT NV, et al. Incidence and mortality of bone cancer among children, adolescents and young adults of Brazil. Clinics, 2019; 74: 1-8

4. BAUER DFV, et al. Crianças com câncer: caracterização das internações em um hospital escola público. Semina: Ciências Biológicas e da Saúde, 2016; 36(1): 9-16.

5. BOUZAS LF, CALAZANS M. Tumores sólidos e hematológicos na infância e na adolescência-Parte 2. Adolescencia e Saude, 2007; 4(2): 12-18.

6. BRAGA PE, et al. Câncer na infância: análise comparativa da incidência, mortalidade e sobrevida em Goiânia (Brasil) e outros países. Cadernos de Saúde Pública, 2002; 18(1): 33-44.

7. BRAVO LE, et al. Descriptive epidemiology of childhood cancer in Cali: Colombia 1977-2011. Colombia Médica, 2013; 44(3): 155.

8. CARDOUS-UBBINK MC, et al. Risk of second malignancies in long-term survivors of childhood cancer. European Journal of Cancer, 2007; 43: 351-362.

9. CURVO HRM, et al. Morbidity and mortality from cancer children and adolescents associated with the agricultural use of pesticides in the state of Mato Grosso, Brazil. Cadernos Saúde Coletiva, 2013; 21(1): 10-17.

10. DINIZ AB, et al. Perfil epidemiológico do câncer infantil na população atendida por uma unidade de oncologia pediátrica em Salvador-Bahia. Revista de Ciências Médicas e Biológicas, 2005; 4: 131-139. 
11. FRANCO EL. Cancer epidemiology: substance and methods. Cienc. Cult. (São Paulo), 1994; 46(1/2): 46-62.

12. FREITAS VA, et al. Distribuiçao proporcional dos tipos histológicos mais frequentes em crianças no hospital de câncer do mato grosso no período de 2007-2011. Seminário Transdisciplinar da Saúde, 2013; 7.

13. FRIESTINO JKO. Panorama do câncer em crianças e adolescentes sob a perspectiva da saúde coletiva. Revista baiana saúde pública, 2017; 40: 2 .

14. HADAS TC, et al. Câncer pediátrico: perfil epidemiológico dos pacientes atendidos no serviço de oncologia pediátrica do hospital de clínicas da UFPR. Revista Médica da UFPR, 2014; 1(4): 141-9.

15. HANNA N, et al. Systemic therapy for stage IV non-small-cell lung cancer: American Society of Clinical Oncology clinical practice guideline update. Journal of Clinical Oncology, 2017; 35(30): 3484-3515.

16. HIYAMA E. Pediatric hepatoblastoma: diagnosis and treatment. Translational pediatrics, 2014; 3(4): 293.

17. LUCENA JN, et al. Aspectos clínicos, epidemiológicos e sobrevida de crianças com neuroblastoma: 21 Anos de experiência do instituto de oncologia pediátrica, São Paulo. Revista Paulista de Pediatria, 2018; 36(3): 254-260.

18. MARCHI JA, et al. Câncer infanto juvenil: perfil de óbitos. Revista da Rede de Enfermagem do Nordeste, 2013; 14(5): 911-919.

19. MARQUANT F, et al. Risk of Childhood Cancer and Socio- economic Disparities: Results of the French Nationwide Study Geocap 2002-2010. Paediatric and perinatal epidemiology 2016; 30(6): 612-622.

20. MARTINS SMG, LIMA TNST. Morbimortalidade dos três principais tipos de câncer infantojuvenil em sergipe (20132018). (Mestrado em Saúde e Ambiente) - Instituto de Tecnologia e Pesquivsa. Universidade Tiradentes, Aracaju; 2019.

21. MONTE O, et al. Carcinoma de tireoide na infância e adolescência. Arquivos brasileiros de endocrinologia Metabólica 2007; 51(5): 763-768.

22. PEDROSA M, et al. Non-Hodgkin's lymphoma in childhood: clinical and epidemiological characteristics and survival analysis at a single center in Northeast Brazil. Jornal de pediatria, 2007; 83(6): 547-554.

23. PESOLA F, et al. Cancer incidence in English children, adolescents and young people: past trends and projections to 2030. British journal of cancer, 2017; 117(12): 1865.

24. RONDINELLI PI, et al. Tumores de células germinativas intracranianos na infância: avaliação de 14 casos. Arquivos de Neuropsiquiatria, 2005; 63(3B): 832- 836.

25. SANTOS-JÚNIOR CJ, et al. Características clínico-epidemiológicas do câncer infantojuvenil no estado de Alagoas, Brasil. Revista de Medicina, 2018; 97(5), 454- 460.

26. SIEGEL DA, et al. Taxas e tendências de incidência de câncer entre crianças e adolescentes nos Estados Unidos, 2001-2009. Pediatrics, 2014; 134 (4): 945-955.

27. SOUZA MHG, et al.. SARCOMA DE EWING: DIAGNÓSTICO PRECOCE DO CÂNCER PEDIÁTRICO. Revista Brasileira Interdisciplinar de Saúde, 2019; 1(3): 1.

28. STELIAROVA-FOUCHER E, et al. Changing geographical patterns and trends in cancer incidence in children and adolescents in Europe, 1991-2010 (Automated Childhood Cancer Information System): a population-based study. The Lancet Oncology, 2018; 19(9): 1159- 1169.

29. TEIXEIRA AB. Avaliação do perfil epidemiológico das crianças internadas no setor de oncologia pediátrica do Hospital da Criança em Feira de Santana. Anais Seminário de Iniciação Científica 2017; 21(2): 1-3.

30. WARLET F, et al. Neuroblastoma infantil: características clínicas, tratamento e prognóstico. Acta médica, 2018; 39(2): 259-268. 